\title{
Measurement of the Tau Lepton Lifetime with BABAR
}

\author{
Alberto Lusiani ${ }^{\mathrm{a}}$ \\ for the BaBar collaboration

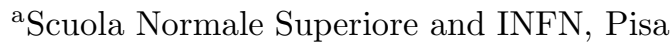

The mean lifetime of the tau lepton is measured from the decay length distribution of 3-prong tau decays from $e^{+} e^{-}$collisions at the $\Upsilon(4 S)$ resonance. A data sample of $80.0 \mathrm{fb}^{-1}$ collected with the BABAR detector at the PEP-II B Factory is used for this measurement. The measured tau lifetime is: $\tau_{\tau}=289.40 \pm 0.91$ (stat.) \pm 0.90 (syst.) fs. All the results are preliminary.

\section{INTRODUCTION}

When combined with measurements of the tau mass and branching ratios, the tau lifetime provides a precise test of the lepton universality hypothesis [1], which is assumed in the Standard Model. In the following, we describe a measurement of the tau lifetime with the BABAR experiment, that reaches a higher precision than measurements at LEP [2]. The BABAR environment differs significantly from that of LEP in that the tau energy is smaller and the center-of-mass reference frame is not at rest in the laboratory. This precision measurement, therefore, has systematic uncertainties quite different from the LEP experiments. Special attention has been devoted to a careful study of systematic uncertainties related to the detector alignment. To avoid experimenter's bias, a blinded [3] analysis procedure was adopted, with the mean decay length remaining unknown until all systematic biases and errors were finalized and all cross checks were performed.

\section{DETECTOR AND DATASET}

The data used in this analysis were collected with the BABAR detector at the PEP-II storage ring at the $\Upsilon(4 S)$ resonance and correspond to an integrated luminosity of $80.0 \mathrm{fb}^{-1}$. With a cross section for tau pairs at $\sqrt{s}=10.58 \mathrm{GeV}$ of $\sigma_{\tau \tau}=(0.89 \pm 0.02) \mathrm{nb}$ [4], this data sample contains 71.2 million $e^{+} e^{-} \rightarrow \tau^{+} \tau^{-}$events. The collider is operated with asymmetric beam ener- gies, producing a boost of $\beta \gamma \approx 0.56$ along the collision axis.

The BABAR detector is described elsewhere [5]. Charged-particle (track) trajectories and momenta are measured with a 5-layer double-sided silicon vertex tracker and a 40-layer drift chamber inside a 1.5- $\mathrm{T}$ superconducting solenoidal magnet. BABAR achieves an impact parameter resolution of about $40 \mu \mathrm{m}$ for charged particles with momenta higher than $1 \mathrm{GeV} / c$. An electromagnetic calorimeter (EMC) consisting of 6580 Thalliumdoped CsI crystals is used to identify electrons and photons. A ring-imaging Cherenkov detector is used to identify charged hadrons, and the instrumented magnetic flux return (IFR) is used to identify muons.

A GEANT 4-based[6] Monte Carlo (MC) simulation is used to model the signal and part of the expected background events. Samples equivalent to approximately two times the accumulated data were used to model $e^{+} e^{-} \rightarrow \tau^{+} \tau^{-}, u \bar{u}, d \bar{d}, s \bar{s}$, and $c \bar{c}$ events, and samples equivalent to approximately four times the accumulated data were used to model $B \bar{B}$ events.

\section{ANALYSIS METHOD}

This measurement is based on a high purity sample, sacrificing efficiency in order to reduce background contributions and retain only well measured events. The measurement bias and the background contamination from hadronic events are estimated with a Monte Carlo simulation, with checks on data. Backgrounds from two- 
photon and radiative Bhabha events are studied and estimated on data.

In the following, unless otherwise noted, all particles attributes are evaluated in the $e^{+} e^{-}$ center-of-mass (CM) frame. Charged particles momenta are boosted from the laboratory (LAB) frame to the CM frame assuming that they have the pion mass. Photon candidates are reconstructed from neutral EMC clusters with a deposited energy above $100 \mathrm{MeV}$ that are not associated to tracks. Their 4-momenta are set (and boosted) assuming they are photons that originate from the nominal interaction point.

\subsection{Candidate Selection}

Candidate signal events are required to have a "1-3 topology", where one tau decay yields three charged particles (3-prong), while the second tau decay yields one charged particle (1prong). Therefore, four tracks with zero net charge are required, pointing to the luminous region, as expected for decay products of a short lived particle. The opening angle between the highest momentum track and each of the remaining three tracks must be larger than $90^{\circ}$. The plane perpendicular to the highest momentum track divides the surviving events into two hemispheres: one hemisphere contains one track while the other contains exactly three, defining the 13 topology. In the 3-prong hemisphere, all three tracks must have at least 6 hits in the SVT, and all pairs of oppositely charged tracks must have an $e^{+} e^{-}$invariant mass above $150 \mathrm{MeV} / c^{2}$, in order to reject radiative Bhabha events. In order to remove events at the edge of the detector acceptance, the cosine of the polar angle of the 3-prong tracks total momentum vector is required to be between -0.9 and 0.8 .

The 1-prong track must be identified as an electron candidate, according to the ratio of the energy detected in the EMC to the track momentum, the cluster shape in the EMC, the energy loss in the DCH, and the DIRC Cherenkov angle, if available. On the other side, all 3-prong tracks must fail the electron identification criteria. In order to select the $\tau^{-} \rightarrow e^{-} \bar{\nu}_{e} \nu_{\tau}$ decay mode $^{1}$, the 1 -prong hemisphere must not contain

\footnotetext{
${ }^{1}$ Throughout this paper, charge conjugate decay modes
}

any photon candidates.

To reduce background further, several requirements on the event's kinematic properties are imposed:

- The event thrust computed using the four tracks must be larger than 0.92 (to suppress hadronic and two-photon background) and smaller than 0.995 (to suppress Bhabha events).

- The energy of the four tracks ("charged energy") is required to be larger than $4 \mathrm{GeV}$ in order to reject two-photon events.

- We define the visible energy as the sum of the energies of the photon candidates and charged particle candidates. The missing energy is computed by subtracting the visible energy from the event CM energy, determined from the beam energies, and is required to be larger than $1.5 \mathrm{GeV}$, in order to account for undetected neutrinos in tau decays.

- The ratio $\sin \alpha=p_{\mathrm{T}} /\left(E_{\mathrm{CM}}-E_{\mathrm{CH}}\right)$, where $p_{\mathrm{T}}$ is the net transverse momentum of the four tracks, $E_{\mathrm{CM}}$ is the event CM energy, and $E_{\mathrm{CH}}$ is the charged energy, must be larger than 0.05 .

- The momentum of the 1-prong track must be between $1.72 \mathrm{GeV} / c$ and $4.25 \mathrm{GeV} / c$, in order to suppress two-photon and Bhabha events, which are expected to populate the low and high momenta regions of the 1prong momentum distribution, respectively.

Although Bhabha and two-photon events are not included in the Monte Carlo samples, the simulation predicts the number of selected candidates with an accuracy better than $5 \%$. The amount of Bhabha and two-photon events in the selection has been estimated to be lower than $1 \%$ (see Section 4).

\subsection{Tau Decay Length}

The tau decay length is reconstructed in the plane transverse to the boost direction ("transverse plane"). The tau production point is taken

also are implied. 
to be the center of the luminous region, projected onto the transverse plane. The decay vertex is determined from a $\chi^{2}$ fit to the tracks in the transverse plane.

The tau decay length in the transverse plane $\left(\lambda_{t}\right)$ is reconstructed by projecting the tau decay flight from the tau production point $\left(\bar{x}_{p}\right)$ to the decay point $\left(\bar{x}_{d}\right)$ onto the estimated tau flight direction, represented by the unit vector of the 3 prong total momentum in the transverse plane $\left(\widehat{p}_{t}\right)$ :

$\lambda_{t}=\left(\bar{x}_{p}-\bar{x}_{d}\right) \cdot \widehat{p}_{t}$.

The tau decay length is reconstructed from the transverse tau decay length and the sine of the tau polar angle with respect to the boost direction $(\sin \theta)$, where the direction of the tau flight is taken to be the direction of the 3-prong total momentum vector:

$\lambda=\frac{\lambda_{t}}{\sin \theta}$.

The uncertainty in the reconstructed decay length is estimated including contributions from the uncertainties on the tau production and decay points on the transverse plane. The uncertainty on the tau production point comes from the spatial distribution of collision points, which is measured on a run-by-run basis using two-prong events. The distribution is parameterized as a multi-variate Gaussian, with a width corresponding to $\sigma_{x} \approx 120 \mu \mathrm{m}$ and $\sigma_{y} \approx 5 \mu \mathrm{m}$ on the horizontal and vertical plane, respectively. The uncertainty on the decay vertex is determined by the vertex fit from the uncertainties on the track parameters. The uncertainty associated with approximating the true direction of the tau with that obtained using the 3 -prong vector momentum has been studied and found to be negligible for this analysis $(\approx 4 \%)$.

The rms width of the pull distribution is 1.07: the deviation from unity is statistically significant and indicates that the estimated tracking errors are underestimated by $7 \%$ in Monte Carlo. The distribution of the difference between the reconstructed and the true decay length has a rms width of $327 \mu \mathrm{m}$ and a mean of $(0.85 \pm 0.42) \mu \mathrm{m}$. For comparison, the input Monte Carlo mean tau decay length is $240 \mu \mathrm{m}$. The mean differs from zero by $2.0 \sigma$. Systematic effects related to the observed pull and residual distributions are discussed in Section 4.1. The distribution of the reconstructed decay length is shown in Fig. 1.

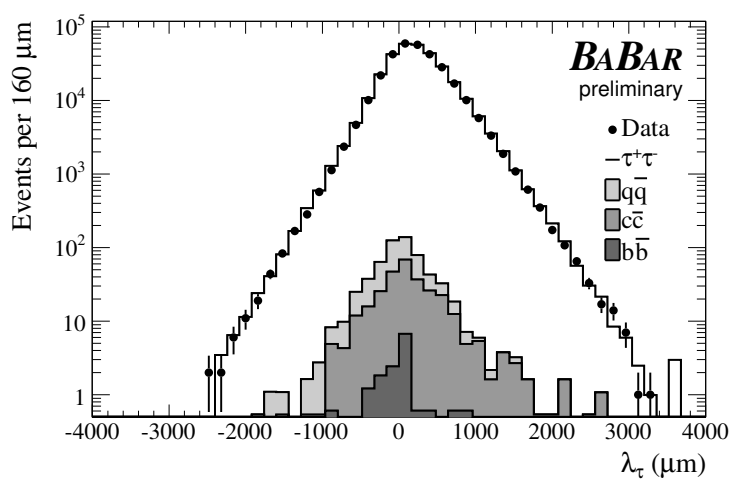

Figure 1. Distribution of the decay length for real and simulated events.

To insure the quality of the decay length reconstruction, the following requirements are imposed:

- The 3-prong tracks are fitted to a common vertex in the laboratory frame, using the full covariance error matrix; the fit $\chi^{2}$ probability is required to be larger than $1 \%$.

- In order to retain only relatively wellmeasured events, the estimated decay length uncertainty is required to be smaller than $700 \mu \mathrm{m}$.

- Reconstructed decay lengths on the extreme negative and positive tails of the distribution are removed, provided their absolute value is larger than $1000 \mu \mathrm{m}$, when the probability of measuring their value and all values further away in the respective tail is smaller than $1 \cdot 10^{-5}$. For this purpose, the expected tau decay length distribution 
is computed as an event-by-event Gaussiansmeared decaying exponential with the decay constant set to the 2002 world average tau lifetime [7] and the Gaussian smearing set to the estimated decay length uncertainty.

Including the above requirements, the number of surviving data candidates is about 312,000 ; the simulation predicts $317,000 \tau^{+} \tau^{-}$events, with a contamination of about $600 u \bar{u}, d \bar{d}, s \bar{s}, c \bar{c}$ and $B \bar{B}$ events. According to Monte Carlo, $0.44 \%$ of the produced $\tau^{+} \tau^{-}$events satisfy our selection criteria (7.78\% of $\tau^{+} \rightarrow e^{-} X, \tau^{-} \rightarrow 3$-prongs $X^{\prime}$, according to the known branching fractions[7]).

\subsection{Tau Mean Lifetime}

In order to be less sensitive to the modeling of the estimated uncertainty, the mean decay length is computed with an unweighted average. If the estimated errors on the decay length were used, the statistical uncertainty on the mean would decrease by only $\approx 13 \%$, due to the contribution from the natural width of the exponential decay.

In order to decrease the sensitivity to the beam spot position and size and to the detector alignment, the averaging is first performed separately in 60 intervals of the 3-prong momentum azimuthal angle, and the final average is computed as the unweighted average of the 60 bin-averages. This procedure improves the effective uniformity of the azimuthal occupancy. The statistical error on the averaged decay length is determined by measuring the decay length variance when repeating the measurement on 100 sub-samples of the selected candidates ${ }^{2}$ and amounts to $\sigma\left(\lambda_{\tau}\right)=$ $0.752 \mu \mathrm{m}$. In comparison, the error computed from the rms width of the decay length distribution and the number of events is $0.719 \mu \mathrm{m}$.

The mean lifetime is computed from the mean decay length, the mean tau momentum and the tau mass. The mean tau momentum is estimated using simulated events that are produced at the same energy as the data. The statistical error is set to the mean decay length uncertainty; uncertainties on the tau average momentum and mass

${ }^{2}$ The selected candidates are ordered according to the time of their acquisition, and divided in 100 blocks containing an approximately equal number of events. are accounted for in the systematic error. The mean lifetime measurement on the data candidates includes biases due to the measurement procedure and to backgrounds: these are discussed in the next section.

\section{SYSTEMATIC UNCERTAINTIES}

A number of systematic biases and uncertainties are estimated, including effects coming from the measurement procedure, from background contamination, and from the detector alignment and length scale.

\subsection{Measurement Bias}

The bias due to the measurement procedure is determined from the difference between the mean lifetime measured on the signal Monte Carlo sample and the Monte Carlo input lifetime. The bias uncertainty is determined by the Monte Carlo lifetime measurement statistical error. Several additional systematic uncertainties related to the simulation of the measurement bias are investigated, and all are found to be negligible in comparison.

On simulated events, the measurement bias can be decomposed into contributions coming from the selection bias and from the reconstruction of the decay length and of the tau momentum (Table 1) by sequentially replacing reconstructed quantities with true ones in the reconstruction sequence. The bias on the decay length reconstruction can be further decomposed into contributions from the reconstruction of the tau polar angle, of the tau azimuthal angle, and of the decay length on the true tau flight direction (Table 1).

The bias from the selection criteria is compatible with zero. The bias related to the tau momentum reconstruction is also compatible with zero, as expected since the simulated mean tau momentum is used for simulated events: systematic uncertainties arising from using the simulated mean tau momentum for data are discussed in Section 4.4. Uncertainties on the tau direction reconstruction using only charged particle candidates are primarily determined by the momentum distribution of neutrinos and neutral particles in 3-prong tau decays; uncertainties related to the 
simulation of tracking resolution and of the tau branching fractions are found to be negligible.

The bias on the decay length reconstruction along the true tau direction $(\approx 1 \%)$ is determined primarily by correlations in $d_{0}$ and $\phi_{0}$ tracking errors due to scattering of the charged tau decay products in the detector [8]. The related bias on the measured decay length $\lambda$ is expected to be of the order of $\Delta \lambda / \lambda \approx \gamma /\left(\beta c \tau_{\tau}\right) \cdot\left\langle\Delta d_{0} \Delta \phi_{0}\right\rangle$ [8], where $\beta$ and $\gamma=1 / \sqrt{1-\beta^{2}}$ describe the tau boost in the laboratory frame, and $\Delta d_{0}, \Delta \phi_{0}$ are the reconstruction residuals on the track helix parameters $d_{0}, \phi_{0}$. Expected uncertainties on $d_{0}$ and $\phi_{0}$ have very similar distributions for real and simulated events, and the average of the corresponding terms of the tracking parameters' covariance matrix for real and simulated 3 -prong tracks differs by $(2.5 \pm 1.1) \%$. This corresponds to a negligible variation of the measurement bias. From a comparison of the 3-prong vertex fit $\chi^{2}$, the simulation is found to reproduce the data tracking residuals with a relative bias of $(2.3 \pm 0.2) \%$. This corresponds to a negligible uncertainty on the measurement bias.

The estimated measurement bias is affected by imperfections of the simulation in reproducing the data event properties. To estimate the related uncertainties, Monte Carlo events are weighted to match the data distributions of the cosine of the polar angle, of the 1-prong momentum, of the 3-prong total momentum, of the decay length estimated uncertainty, of the sum of the sines of the track-to-track opening angles among the 3prong tracks, of the $d_{0}-\phi_{0}$ tracking error correlations, and of the $\chi^{2}$ of the 3-prong vertex fit in the transverse plane. The corresponding operation is also performed on data events. In all cases, the variation of the measurement bias is negligible ( $\leq 0.03 \%$ of the mean lifetime).

It has been checked on simulated events that when changing the tau decay rates to $K^{-}$and $K_{S}^{0}$, the variation of the bias is negligible in comparison to the uncertainty $(0.22 \%)$ coming from Monte Carlo. The lifetime measurement on data is assumed to have the same relative bias that is measured on Monte Carlo, and is consequently subtracted. The validity of this procedure has been verified on Monte Carlo samples that simulate different input tau lifetimes by weighting events.

Since we have found no evidence for additional non negligible uncertainties, the Monte Carlo statistical error is taken as the overall systematic uncertainty on the measurement bias.

Table 1

Measurement bias contributions. The quoted uncertainties are statistical only. Because of eventby-event correlations among the individual biases, they do not add up exactly to the resulting total bias.

\begin{tabular}{lr}
\hline \multicolumn{1}{c}{ bias contribution } & \multicolumn{1}{c}{ bias $(\%)$} \\
\hline total measurement bias & $0.336 \pm 0.220$ \\
- selection & $-0.029 \pm 0.127$ \\
- tau momentum & $0.016 \pm 0.010$ \\
- decay length reconstr. & $0.350 \pm 0.179$ \\
- tau $\theta$ angle reconstr. & $-0.529 \pm 0.007$ \\
- tau $\phi$ angle reconstr. & $-0.666 \pm 0.001$ \\
- reconstruction & $1.295 \pm 0.170$ \\
\hline
\end{tabular}

\subsection{Background}

For each expected background sample, a systematic bias and uncertainty is computed. The uncertainty includes contributions from uncertainties on the expected contamination level and decay length distribution of the expected backgrounds, and from the Poissonian fluctuations due to the finite number of the expected background events.

The bias due to $u \bar{u}, d \bar{d}, s \bar{s}, c \bar{c}$ and $B \bar{B}$ events is measured from the difference between the combined Monte Carlo and the signal Monte Carlo mean lifetime. The accuracy of the simulation in predicting the hadronic background is checked for events with large 3-prong invariant masses $\left(M_{3}>1.6 \mathrm{GeV} / c^{2}\right)$, where hadronic background prevails over the $\tau^{+} \tau^{-}$events; furthermore, uncertainties on the simulation of hadron rejection in electron identification are estimated on data, using different selectors, whose hadron rejection 
has been measured on data control samples. After all checks on data, the uncertainty on the Monte Carlo prediction of the hadronic contamination is set to $100 \%$.

The background contamination from twophoton events is determined by studying the charged energy distribution for real and simulated events. The charged energy distribution of twophoton events is well modeled with an exponential $f(E)=\exp \left(-E / E_{\gamma \gamma}\right)$, with $E_{\gamma \gamma} \approx 0.6 \mathrm{GeV}$, on a control data sample whose content of twophoton events is greatly enhanced by inverting the selection requirements on the 1-prong electron identification, on $\sin \alpha$, on the event thrust, and on the minimum 1-prong momentum. An upper limit to the background contamination from twophoton events is determined by fitting the excess of real over simulated events on the charged energy distribution, releasing the selection requirements on the minimum charged energy itself, on the minimum event thrust, on $\sin \alpha$, and on the minimum 1-prong momentum. In this fit, the shape of the charged energy distribution of the two-photon events contribution is fixed with a fit to the greatly enhanced control sample. The relative normalization of simulated over real events is left free in the fit, in order to include uncertainties on the data efficiency simulation, which are set to $5 \%$. The two-photon contamination is taken to be zero with a systematic uncertainty corresponding to estimated upper limit. The average and the spread of the decay length distribution of the two-photon background events are determined from a data sample enhanced in twophoton events by selecting data candidates for which the charged energy is required to be between $3 \mathrm{GeV}$ and $5 \mathrm{GeV}$, the $\sin \alpha$ requirement is inverted, the minimum event thrust requirement is released, and the minimum 1-prong momentum requirement is released. The contribution from $\tau^{+} \tau^{-}, u \bar{u}, d \bar{d}, s \bar{s}, c \bar{c}$ and $B \bar{B}$ events to the resulting decay length distribution is subtracted by using simulated events.

The amount of Bhabha background contamination is measured by fitting the discrepancy between real and simulated events on the high end of the 1-prong momentum distribution. The shape of the Bhabha background distribution is adequately fit with an empirical model based on events that fail the veto on identified electrons in the 3-prong tracks, but otherwise satisfy all the other selection requirements. The normalization of the Bhabha contamination distribution in the selected candidates is fit on the selected real and simulated candidates, and $1215 \pm$ 134 (stat.) \pm 31 (syst.) events are predicted below the maximum 1-prong momentum requirement. The relative normalization of simulated and real events is left free in the fit, as for the twophoton background estimate. The systematic uncertainty on the Bhabha contamination level is determined from the uncertainties on the shape of the 1-prong momentum distribution for Bhabha events. The average and the spread of the decay length distribution and the uncertainties on the estimated bias from Bhabha background are computed along the same lines as for the two-photon component.

Table 2 reports the contributions to the background related systematic uncertainty from $u \bar{u}$, $d \bar{d}, s \bar{s}, c \bar{c}, B \bar{B}$, Bhabha and two-photon events.

Table 2

Background biases and uncertainties on the decay length measurement.

\begin{tabular}{lr}
\hline background & \multicolumn{1}{c}{$\Delta \lambda_{\tau}(\%)$} \\
\hline$u \bar{u}, d \bar{d}, s \bar{s}$ & $-0.104 \pm 0.113$ \\
$c \bar{c}$ & $-0.025 \pm 0.045$ \\
$B \bar{B}$ & $-0.004 \pm 0.007$ \\
two-photon & $0.000 \pm 0.030$ \\
Bhabha & $-0.295 \pm 0.066$ \\
Total & $-0.428 \pm 0.142$ \\
\hline
\end{tabular}

\subsection{Detector Alignment}

Track properties at the tau production and decay points are primarily determined by the SVT, whose local alignment has been determined using $e^{+} e^{-}$collisions and cosmic ray data, and with optical surveys. The alignment procedure also includes information from optical surveys on the SVT wafers, which measured the wafers' linear 
dimensions, hence also the average strip spacing, with a precision of $0.01 \%$. The uncertainties on the alignment parameters are confirmed by studies of the performance of the BABAR tracking system on data events. Uncertainties using SVT alignment are modeled on simulated data by changing the wafer positions and orientations with respect to a perfectly aligned detector according to six reference alignment sets, which model the detector alignment uncertainties, including the uncertainties on the SVT wafers' radial positions and on the overall radial length scale of the BABAR tracking system. The global length scale of BABAR tracking along the beam direction $\left(s_{z}\right)$ has been independently measured with studies of the $z$ distribution of vertices reconstructed from particles coming from interactions of off-beam electrons with the beam pipe material, and amounts to $s_{z}=(100.04 \pm 0.11) \%$. Alignment related uncertainties are estimated by re-fitting tracks belonging to about 72,000 signal Monte Carlo events using first a perfectly aligned detector and then the six above mentioned reference alignment sets. For each alignment set, the reconstructed decay length shift with respect to a perfect alignment is plotted. From these distributions, one measures six mean decay length biases, with uncertainties that are determined from the respective spread and number of events. The resulting biases are statistically compatible with zero, and an overall systematic uncertainty due to detector alignment is computed by summing in quadrature all the uncertainties: this amounts to $0.10 \%$. Finally, a contribution due to the uncertainty on the $z$ length scale is added to this number, with a weight related to the average polar angle of the selected candidates, in order to obtain the total systematic uncertainty due to the $B A B A R$ length scale and alignment, which is $0.11 \%$.

\subsection{Other Systematic Errors}

The distribution of the collisions in the luminous region is measured on data and is parametrized as a multi-variate Gaussian probability distribution, whose principal axes are along the boost direction $z$, the vertical direction $y$, and the horizontal direction $x$ perpendicular to the other two axes. The beam spot parameters are varied by their uncertainties, and summing all biases in quadrature yields a total systematic uncertainty of $0.04 \%$.

Uncertainties on the beam energies correspond to uncertainties on the $\mathrm{CM}$ event energy and boost. The uncertainty on the CM energy $(2 \mathrm{MeV})$ corresponds to a systematic contribution of $0.043 \%$. According to studies on di-lepton events, the uncertainty on the event momentum size is $7 \mathrm{MeV} / c$, and the uncertainty on its transverse component is $1.1 \mathrm{MeV} / c$. The corresponding uncertainties on the boost size and direction add systematic contributions equal to $0.003 \%$ and $0.003 \%$, respectively. These contributions are combined assuming no correlation.

The tau mass has been set to the current world average [9] value and varied by its uncertainty to estimate its systematic effect. According to the Monte Carlo, which is based on the KoralB generator [10], radiative events cause an average $2 \%$ loss with respect to the maximum allowed tau momentum. The uncertainty of this prediction is conservatively set to $0.10 \%$. This estimate is confirmed by comparing the KoralB generator (which simulates QED radiation up to order $\alpha$ ) with KK2f [11] (order $\alpha^{2}$ ), and by studies on $\mu^{+} \mu^{-} \gamma$ events on data.

Finally, we have verified that the Monte Carlo simulation correctly models how the mean decay length varies when changing the selection requirements on the 3 -prong vertex $\chi^{2}$ probability, on the maximum estimated decay length uncertainty, and the removal of events with large and improbable reconstructed decay lengths.

\subsection{Summary of Systematics}

Table 3 lists the systematic biases and uncertainties on the tau lifetime measurement.

\section{PHYSICS RESULTS}

After subtraction of the estimated biases, the tau lifetime is measured to be:

$\tau_{\tau}=289.40 \pm 0.91$ (stat.) \pm 0.90 (syst.) fs.

The asymmetry in the lifetime of negative and positive tau leptons, $\tau_{\tau-}$ and $\tau_{\tau+}$, is measured to 
Table 3

Systematic biases and uncertainties.

\begin{tabular}{lr}
\hline Systematic uncertainty source & $\Delta \tau_{\tau}(\%)$ \\
\hline Measurement bias & $0.336 \pm 0.220$ \\
Background & $-0.428 \pm 0.142$ \\
alignment and length scale & \pm 0.111 \\
beam spot position & \pm 0.043 \\
beam spot size & \pm 0.044 \\
beam energies and direction & \pm 0.043 \\
$\tau$ mass & \pm 0.006 \\
$\tau$ momentum & \pm 0.100 \\
Total & $-0.092 \pm 0.310$ \\
\hline
\end{tabular}

be:

$\frac{\tau_{\tau-}-\tau_{\tau+}}{\tau_{\tau-}+\tau_{\tau+}}=[0.12 \pm 0.32] \%$,

which represents a test of the validity of the CPT theorem in the tau lepton decay. On this result, no dedicated systematic studies have been done yet. All results are preliminary.

This measurement, combined with the world average tau lifetime [9] $\left(\tau_{\tau}^{\mathrm{WA}}=290.6 \pm 1.1 \mathrm{fs}\right)$ gives:

$\tau_{\tau}=290.09 \pm 0.83 \mathrm{fs}$,

assuming no correlation between the two systematic uncertainties. Using the 2004 [9] world averages for the other particle properties, and combining the tau branching fraction measurements into electron and muon assuming $e-\mu$ universality, this measurement is compatible with the lepton universality assumption, in the Standard Model framework (Fig. 2).

\section{REFERENCES}

1. Y.S. Tsai, Phys. Rev. D4, 2821 (1971);

H.B. Thacker and J.J. Sakurai, Phys. Lett. B36, (1971) 103.

2. The OPAL Collaboration, G. Alexander et al., Phys. Lett. B374, (1996) 341;

The L3 Collaboration, M. Acciarri et al., Phys. Lett. B479, (2000) 67;

The ALEPH Collaboration, R. Barate et al.,

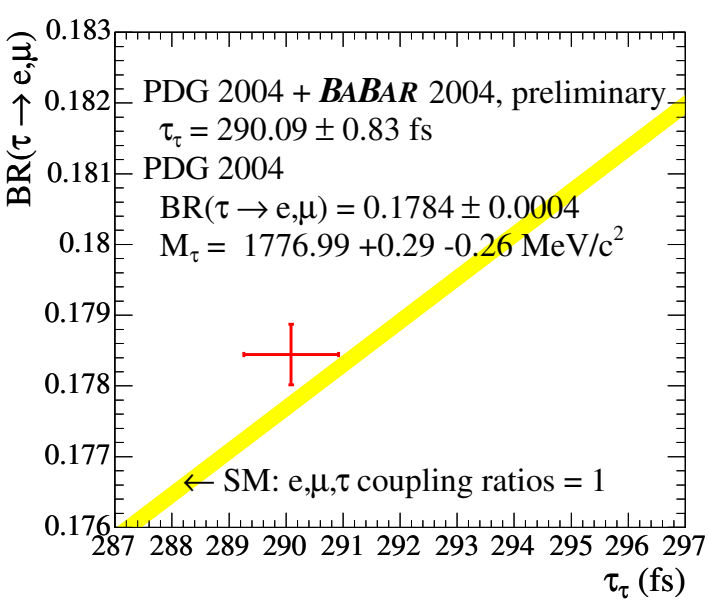

Figure 2. Standard model prediction in the plane of the tau leptonic branching fraction vs. the tau lifetime.

Phys. Lett. B414, (1997) 362;

The DELPHI Collaboration, P. Abreu et al., Phys. Lett. B365, (1996) 448.

3. P. F. Harrison, J. Phys. G28, (2002) 2679.

4. B. F. Ward, S. Jadach, and Z. Was, Nucl. Phys. Proc. Suppl. 116, (2003) 73.

5. The BABAR Collaboration, B. Aubert et al., Nucl. Instrum. Methods A479, (2002) 1.

6. Geant4 Collaboration, K. Amako et al., Nucl. Instr. Meth. A 453, (2000) 455.

7. Particle Data Group, K. Hagiwara et al., Phys. Rev. D66, (2002) 010001.

8. S. Wasserbaech, Phys. Rev. D 48, (1993) 4216.

9. Particle Data Group, S. Eidelman et al., Phys. Lett. B592, (2004) 1.

10. S. Jadach and Z. Was, Comput. Phys. Commun. 85, (1995) 453.

11. S. Jadach, B.F.L. Ward, and Z. Was, Comput. Phys. Commun. 130, (2000) 260. 